Supporting Information

\title{
Controlling Open-Circuit Voltage in Organic Solar Cells by Terminal Fluoro-Functionalization of Narrow-Bandgap $\pi$-Conjugated Molecules
}

Seiichi Furukawa, ${ }^{\dagger, \S}$ Hideaki Komiyama, ${ }^{\S}, \AA$ and Takuma Yasuda ${ }^{*},, \S$

†Department of Applied Chemistry, Graduate School of Engineering,

${ }^{\S}$ INAMORI Frontier Research Center (IFRC), and

International Institute for Carbon-Neutral Energy Research (WPI-I2CNER),

Kyushu University, 744 Motooka, Nishi-ku Fukuoka 819-0395, Japan

*E-mail: yasuda@ifrc.kyushu-u.ac.jp

Table of Contents:

$\begin{array}{ll}\text { Instrumentation } & \mathrm{S} 2\end{array}$

$\begin{array}{ll}\text { Materials } & \text { S2 }\end{array}$

$\begin{array}{lr}\text { OSC device fabrication and measurements } & \text { S3 }\end{array}$

$\begin{array}{lr}\text { Synthesis and characterization } & \text { S4-8 }\end{array}$

Scheme S1. Synthesis routes for $\mathbf{1 - 4} \quad$ S4

Figure S1. ${ }^{1} \mathrm{H}$ and ${ }^{13} \mathrm{C}\left\{{ }^{1} \mathrm{H}\right\}$ NMR spectra of $\mathbf{1}$ in $\mathrm{CDCl}_{3}$

Figure S2. Photoelectron yield spectra of thin films of 1-4 and BDT-DPP $\quad$ S10

Figure S3. UV-vis absorption spectra of $\mathbf{1 - 4}$ blended with $\mathrm{PC}_{71} \mathrm{BM} \quad \mathrm{S} 10$

Figure S4. $J-V$ characteristics of OSCs based on 1 and $\mathbf{3}$ with different D/A ratios S11

Table S1. Photovoltaic parameters for OSCs based on $\mathbf{1}$ and $\mathbf{3} \quad$ S11

Figure S5. $J-V$ characteristics of an OSC based on BDT-DPP $\quad$ S12

Figure S6. $J-V$ characteristics of hole-only devices using neat film of 1-4 $\quad$ S12 


\section{Instrumentation}

${ }^{1} \mathrm{H}$ and ${ }^{13} \mathrm{C}$ NMR spectra were recorded on a Bruker Avance III 400 spectrometer. Chemical shifts of ${ }^{1} \mathrm{H}$ and ${ }^{13} \mathrm{C}$ NMR signals were quoted to tetramethylsilane $(\delta=0.00)$ and $\mathrm{CDCl}_{3}(\delta=77.0)$ as internal standards. Matrix-assisted laser desorption ionization time-of-flight (MALDI-TOF) mass spectra were collected on a Bruker Daltonics Autoflex III spectrometer using dithranol as the matrix. Elemental analyses were carried out with a Yanaco MT-5 CHN corder. UV-vis absorption spectra were measured with a JASCO V-670Y spectrometer. The HOMO energy levels of the materials in thin films were determined using a Riken-Keiki AC-2 ultraviolet photoelectron spectrometer. The LUMO energy levels were estimated by subtracting the optical energy gap $\left(E_{\mathrm{g}}\right)$ from the measured HOMO energy levels; the $E_{\mathrm{g}}$ values were determined from the onset position of the absorption spectra. Grazing incidence wide-angle X-ray scattering (GIWAXS) patterns were obtained using a BL45XU beamline in Spring-8 with Ni-filtered $\mathrm{CuK} \alpha$ radiation. All quantum chemical calculations were performed using the Gaussian 09 program package. Geometry optimization was carried out using the B3LYP functional with the 6-31G(d) basis set. The lowest excited singlet states were computed using the optimized structures with timedependent density functional theory (TD-DFT) at the same level.

\section{Materials}

Commercially available reagents and solvents were used without further purification unless otherwise noted. All of the reactions were performed under a nitrogen atmosphere in dry solvents using standard Schlenk techniques. Ethanolamine, 2-methoxyethanol, and $\mathrm{Pd}\left(\mathrm{PPh}_{3}\right)_{4}$ were purchased from Sigma-Aldrich. Zinc acetate, 4-fluorophenylboronic acid, 3,5difluorophenylboronic acid, and 3,5-bis(trifluoromethyl)phenylboronic acid were purchased from Wako Pure Chemical Industries. 4-(Trifluoromethyl)phenylboronic acid was purchased from Tokyo Chemical Industry. $\mathrm{PC}_{71} \mathrm{BM}$ was purchased from Frontier Carbon Corporation. PEDOT:PSS (Clevios AI 4083) was purchased from Heraeus. 3-(5-Bromothiophen-2-yl)-6(thiophen-2-yl)-2,5-bis(2-ethylhexyl)-pyrrolo[3,4-c]pyrrole-1,4-dione (5) and 2,6bis(trimethylstannyl)-4,8-bis(5-(2-ethylhexyl)-thiophen-2-yl)benzo[1,2-b:4,5-b']dithiophene (8) were purchased from Suna Tech Inc. 


\section{OSC device fabrication and measurements}

Prepatterned ITO-coated glass substrates (size: $2.5 \times 2.5 \mathrm{~cm}^{2}$ ) were cleaned sequentially by sonicating in detergent solution, deionized water, acetone, and isopropyl alcohol for $10 \mathrm{~min}$ each, and were then subjected to UV/ozone treatment for $15 \mathrm{~min}$. A thin layer $(\sim 30 \mathrm{~nm})$ of $\mathrm{ZnO}$ was prepared by spin-coating (at $5000 \mathrm{rpm}$ for $60 \mathrm{~s}$ ) a precursor solution of zinc acetate $(1.00 \mathrm{~g})$ and ethanolamine $(0.28 \mathrm{~g})$ in 2-methoxyethanol $(10 \mathrm{~mL})$ through a $0.20 \mu \mathrm{m}$ polyethylene membrane filter, followed by baking at $200{ }^{\circ} \mathrm{C}$ for $10 \mathrm{~min}$ under air. The photoactive layer was then deposited by spin-coating (at $1000 \mathrm{rpm}$ for $30 \mathrm{~s}$ ) from a chloroform solution containing a donor material (6.4 $\mathrm{mg} \mathrm{mL} \mathrm{m}^{-1}$ ) and $\mathrm{PC}_{71} \mathrm{BM}\left(9.6 \mathrm{mg} \mathrm{mL} \mathrm{m}^{-1}\right)$, after passing through a $0.45 \mu \mathrm{m}$ poly(tetrafluoroethylene) membrane filter. The thickness of the photoactive layer was 90-140 nm, measured with a Dektak profilometer. Finally, 6-nm-thick $\mathrm{MoO}_{3}$ and 100-nm-thick Ag layers were thermally evaporated on top of the active layer under high vacuum $\left(<5.0 \times 10^{-4} \mathrm{~Pa}\right)$ through a shadow mask defining an active area for each device of $0.04 \mathrm{~cm}^{2}$. The current density-voltage $(J-V)$ and IPCE measurements for the fabricated OSCs were conducted on a computer-controlled Keithley 2400 source measure unit in air under simulated AM 1.5G solar illumination at $100 \mathrm{~mW}$ $\mathrm{cm}^{-2}$ (1 sun) using a Xe lamp-based Bunko-keiki SRO-25 GD solar simulator and IPCE measurement system, calibrated with a standard Si photodiode. 


\section{Synthesis and characterization}

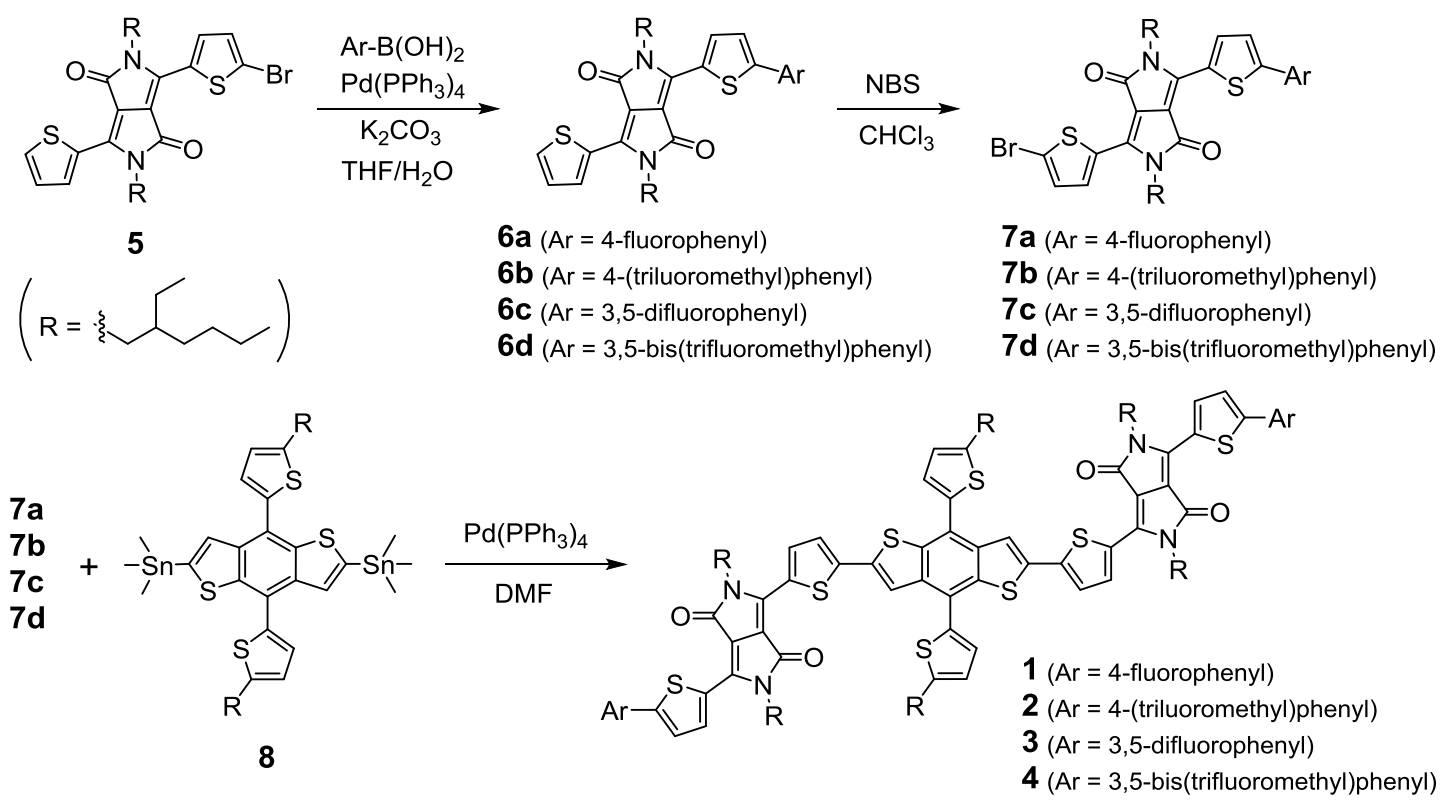

Scheme S1. Synthesis routes for 1-4.

\section{3-\{5-(4-Fluorophenyl)thiophen-2-yl\}-6-(thiophen-2-yl)-2,5-bis(2-ethylhexyl)-pyrrolo}

[3,4-c]pyrrole-1,4-dione (6a). To a mixture of 5 (2.25 g, $3.73 \mathrm{mmol})$ and 4-fluorophenylboronic acid $(0.63 \mathrm{~g}, 4.50 \mathrm{mmol})$ in dry THF $(30 \mathrm{~mL})$ were added $\mathrm{Pd}\left(\mathrm{PPh}_{3}\right)_{4}(0.21 \mathrm{~g}, 0.18 \mathrm{mmol})$ and aqueous $\mathrm{K}_{2} \mathrm{CO}_{3}\left(2.0 \mathrm{M}, 15 \mathrm{~mL} ; \mathrm{N}_{2}\right.$ bubbled before use). The mixture was stirred for $20 \mathrm{~h}$ at $60{ }^{\circ} \mathrm{C}$. After cooling to room temperature, the reaction mixture was added into water and then extracted with chloroform. The combined organic layers were washed with water and dried over anhydrous $\mathrm{Na}_{2} \mathrm{SO}_{4}$. After filtration and evaporation, the product was purified by silica gel column chromatography (eluent: chloroform), recrystallized from chloroform/methanol, and dried under vacuum to afford $\mathbf{6 a}$ as a dark violet solid (yield $=2.07 \mathrm{~g}, 90 \%) .{ }^{1} \mathrm{H} \mathrm{NMR}\left(400 \mathrm{MHz}, \mathrm{CDCl}_{3}\right): \delta$ $8.93(\mathrm{~d}, J=4.3 \mathrm{~Hz}, 1 \mathrm{H}), 8.90(\mathrm{dd}, J=3.9,1.1 \mathrm{~Hz}, 1 \mathrm{H}), 7.68-7.62(\mathrm{~m}, 3 \mathrm{H}), 7.40(\mathrm{~d}, J=4.3 \mathrm{~Hz}$, 1H), 7.28 (m, 1H), 7.17-7.10 (m, 2H), 4.12-4.00 (m, 4H), 1.97-1.83 (m, 2H), 1.44-1.19 (m, 16H), 0.95-0.82 (m, 12H). MS (MALDI-TOF): $\mathrm{m} / z$ calcd $618.27[M]^{+}$; found, 618.00 .

\section{3-[5-\{4-(Trifluoromethyl)phenyl\}thiophen-2-yl]-6-(thiophen-2-yl)-2,5-bis(2-ethylhexyl)-} pyrrolo[3,4-c]pyrrole-1,4-dione (6b). This compound was prepared by a method similar to that of 6a, using 5 (1.52 g, $2.52 \mathrm{mmol})$, 4-(trifluoromethyl)phenylboronic acid (0.53 g, $2.79 \mathrm{mmol})$, 
and $\mathrm{Pd}\left(\mathrm{PPh}_{3}\right)_{4}(0.20 \mathrm{~g}, 0.17 \mathrm{mmol})$. Compound $6 \mathbf{b}$ was obtained as a dark violet solid (yield = $1.57 \mathrm{~g}, 94 \%) .{ }^{1} \mathrm{H}$ NMR (400 MHz, $\left.\mathrm{CDCl}_{3}\right): \delta 8.92(\mathrm{~d}, J=1.0 \mathrm{~Hz}, 1 \mathrm{H}), 8.91(\mathrm{~d}, J=4.3 \mathrm{~Hz}, 1 \mathrm{H})$, $7.78(\mathrm{~d}, J=8.3 \mathrm{~Hz}, 2 \mathrm{H}), 7.68(\mathrm{~d}, J=8.0 \mathrm{~Hz}, 2 \mathrm{H}), 7.64(\mathrm{dd}, J=5.0,1.0 \mathrm{~Hz}, 1 \mathrm{H}), 7.53(\mathrm{~d}, J=4.3$ $\mathrm{Hz}, 1 \mathrm{H}), 7.28(\mathrm{dd}, J=5.0,4.0 \mathrm{~Hz}, 1 \mathrm{H}), 4.11-4.00(\mathrm{~m}, 4 \mathrm{H}), 1.95-1.84(\mathrm{~m}, 2 \mathrm{H}), 1.43-1.19(\mathrm{~m}, 16 \mathrm{H})$, 0.94-0.81 (m, 12H). MS (MALDI-TOF): $\mathrm{m} / z$ calcd $668.27[M]^{+}$; found, 668.02.

\section{3-\{5-(3,5-Difluorophenyl)thiophen-2-yl\}-6-(thiophen-2-yl)-2,5-bis(2-ethylhexyl)-pyrrolo} [3,4-c]pyrrole-1,4-dione (6c). This compound was prepared by a method similar to that of $\mathbf{6 a}$, using 5 (1.52 g, $2.52 \mathrm{mmol}$ ), 3,5-difluorophenylboronic acid (0.47 g, $2.98 \mathrm{mmol})$, and $\mathrm{Pd}\left(\mathrm{PPh}_{3}\right)_{4}$ $(0.11 \mathrm{~g}, 0.10 \mathrm{mmol})$. Compound $\mathbf{6 c}$ was obtained as a dark violet solid (yield $=1.41 \mathrm{~g}, 89 \%) .{ }^{1} \mathrm{H}$ $\operatorname{NMR}\left(400 \mathrm{MHz}, \mathrm{CDCl}_{3}\right): \delta 8.92(\mathrm{~d}, J=1.1 \mathrm{~Hz}, 1 \mathrm{H}), 8.88(\mathrm{~d}, J=4.2 \mathrm{~Hz}, 1 \mathrm{H}), 7.69(\mathrm{dd}, J=5.0$, $1.1 \mathrm{~Hz}, 1 \mathrm{H}), 7.46(\mathrm{~d}, J=4.1 \mathrm{~Hz}, 1 \mathrm{H}), 7.28(\mathrm{dd}, J=5.0,3.9,1 \mathrm{H}), 7.19-7.17(\mathrm{~m}, 2 \mathrm{H}), 6.83-6.79$ (m, 1H), 4.05-3.99 (m, 4H), 1.93-1.83 (m, 2H), 1.43-1.22 (m, 16H), 0.92-0.84 (m, 12H). MS (MALDI-TOF): $\mathrm{m} / \mathrm{z}$ calcd $636.27[M]^{+}$; found, 636.00 .

\section{3-[5-\{3,5-Bis(trifluoromethyl)phenyl\}thiophen-2-yl]-6-(thiophen-2-yl)-2,5-bis(2-}

ethylhexyl)-pyrrolo[3,4-c]pyrrole-1,4-dione (6d). This compound was prepared by a method similar to that of $\mathbf{6 a}$, using 5 (1.52 g, $2.52 \mathrm{mmol})$, 3,5-bis(trifluoromethyl)phenylboronic acid $(0.57 \mathrm{~g}, 2.21 \mathrm{mmol})$, and $\mathrm{Pd}\left(\mathrm{PPh}_{3}\right)_{4}(0.19 \mathrm{~g}, 0.16 \mathrm{mmol})$. Compound $6 \mathbf{d}$ was obtained as a dark violet solid (yield $=1.40 \mathrm{~g}, 95 \%) .{ }^{1} \mathrm{H}$ NMR $\left(400 \mathrm{MHz}, \mathrm{CDCl}_{3}\right): \delta 8.95(\mathrm{~d}, J=2.6 \mathrm{~Hz}, 1 \mathrm{H}), 8.88$ (d, $J=4.4 \mathrm{~Hz}, 1 \mathrm{H}), 8.05(\mathrm{~s}, 1 \mathrm{H}), 7.84(\mathrm{~s}, 1 \mathrm{H}), 7.67(\mathrm{~d}, J=4.0 \mathrm{~Hz}, 1 \mathrm{H}), 7.58(\mathrm{~d}, J=4.0 \mathrm{~Hz}, 1 \mathrm{H})$, $7.30(\mathrm{t}, J=4.0 \mathrm{~Hz}, 1 \mathrm{H}), 4.09-4.03(\mathrm{~m}, 4 \mathrm{H}), 1.92-1.85(\mathrm{~m}, 2 \mathrm{H}), 1.40-1.24(\mathrm{~m}, 16 \mathrm{H}), 0.93-0.85(\mathrm{~m}$, 12H). MS (MALDI-TOF): $\mathrm{m} / \mathrm{z}$ calcd $736.26[M]^{+}$; found, 736.07 .

\section{3-\{5-(4-Fluorophenyl)thiophen-2-yl\}-6-(5-bromothiophen-2-yl)-2,5-bis(2-ethylhexyl)-} pyrrolo[3,4-c]pyrrole-1,4-dione (7a). To a stirred solution of $\mathbf{6 a}(0.73 \mathrm{~g}, 1.18 \mathrm{mmol})$ in dry chloroform (200 mL) was added slowly $N$-bromosuccinimide (NBS, $0.20 \mathrm{~g}, 1.12 \mathrm{mmol}$ ) at $0{ }^{\circ} \mathrm{C}$. The mixture was allowed to warm up to room temperature and stirred overnight. The reaction mixture was poured into water and then extracted with chloroform. The combined organic layers were washed with water and dried over anhydrous $\mathrm{Na}_{2} \mathrm{SO}_{4}$. After filtration and evaporation, the product was purified by recrystallization from chloroform/methanol and dried under vacuum to afford 7a as a dark violet solid (yield $=0.77 \mathrm{~g}, 96 \%) .{ }^{1} \mathrm{H}$ NMR $\left(400 \mathrm{MHz}, \mathrm{CDCl}_{3}\right): \delta 8.95(\mathrm{~d}, J=$ 
$4.0 \mathrm{~Hz}, 1 \mathrm{H}), 8.64(\mathrm{~d}, J=4.3 \mathrm{~Hz}, 1 \mathrm{H}), 7.67-7.62(\mathrm{~m}, 2 \mathrm{H}), 7.40(\mathrm{~d}, J=4.3 \mathrm{~Hz}, 1 \mathrm{H}), 7.24-7.22(1 \mathrm{H})$, 7.16-7.10 (m, 2H), 4.07-4.02 (m, 2H), 3.98-3.92 (m, 2H), 1.98-1.80 (2H), 1.45-1.17 (m, 16H), 0.94-0.81 (m, 12H). MS (MALDI-TOF): $\mathrm{m} / z$ calcd $696.19[M]^{+}$; found, 695.92.

\section{3-[5-\{4-(Trifluoromethyl)phenyl\}thiophen-2-yl]-6-(5-bromothiophen-2-yl)-2,5-bis(2-}

ethylhexyl)-pyrrolo[3,4-c]pyrrole-1,4-dione (7b). This compound was prepared by a method similar to that of 7a, using $\mathbf{6 b}(1.52 \mathrm{~g}, 2.27 \mathrm{mmol})$ and NBS $(0.49 \mathrm{~g}, 2.75 \mathrm{mmol})$. Compound 7b was obtained as a dark violet solid (yield $=0.82 \mathrm{~g}, 48 \%) .{ }^{1} \mathrm{H}$ NMR $\left(400 \mathrm{MHz}, \mathrm{CDCl}_{3}\right): \delta 8.92(\mathrm{~d}$, $J=4.3 \mathrm{~Hz}, 1 \mathrm{H}), 8.66(\mathrm{~d}, J=4.3 \mathrm{~Hz}, 1 \mathrm{H}), 7.77(\mathrm{~d}, J=8.3 \mathrm{~Hz}, 2 \mathrm{H}), 7.68(\mathrm{~d}, J=8.0 \mathrm{~Hz}, 2 \mathrm{H}), 7.53$ (d, $J=4.0 \mathrm{~Hz}, 1 \mathrm{H}), 7.23(\mathrm{~d}, J=4.3 \mathrm{~Hz}, 1 \mathrm{H}), 4.08-4.03(\mathrm{~m}, 2 \mathrm{H}), 3.99-3.93(\mathrm{~m}, 2 \mathrm{H}), 1.94-1.82(\mathrm{~m}$, 2H), 1.43-1.20 (m, 16H), 0.94-0.84 (m, 12H). MS (MALDI-TOF): $\mathrm{m} / z$ calcd $746.18[\mathrm{M}]^{+}$; found, 745.92 .

\section{3-\{5-(3,5-Difluorophenyl)thiophen-2-yl\}-6-(5-bromothiophen-2-yl)-2,5-bis(2-}

ethylhexyl)-pyrrolo [3,4-c]pyrrole-1,4-dione (7c). This compound was prepared by a method similar to that of 7a, using $\mathbf{6 c}(1.13 \mathrm{~g}, 1.77 \mathrm{mmol})$ and NBS $(0.38 \mathrm{~g}, 2.14 \mathrm{mmol})$. Compound 7c was obtained as a dark violet solid (yield $=0.99 \mathrm{~g}, 79 \%)$. ${ }^{1} \mathrm{H} \mathrm{NMR}\left(400 \mathrm{MHz}, \mathrm{CDCl}_{3}\right): \delta 8.89$ (s, 1H), $8.67(\mathrm{~s}, 1 \mathrm{H}), 7.46(\mathrm{~d}, J=4.1 \mathrm{~Hz}, 1 \mathrm{H}), 7.23(\mathrm{~d}, J=4.2 \mathrm{~Hz}, 1 \mathrm{H}), 7.18(\mathrm{~d}, J=6.2 \mathrm{~Hz}, 2 \mathrm{H}), 6.81$ $(\mathrm{t}, J=8.6 \mathrm{~Hz}, 1 \mathrm{H}), 4.01-3.91(\mathrm{~m}, 4 \mathrm{H}), 1.92-1.81(\mathrm{~m}, 2 \mathrm{H}), 1.42-1.25(\mathrm{~m}, 16 \mathrm{H}), 0.92-0.86(\mathrm{~m}, 12 \mathrm{H})$. MS (MALDI-TOF): $\mathrm{m} / z$ calcd $714.18[M]^{+}$; found, 713.86.

\section{3-[5-\{3,5-Bis(trifluoromethyl)phenyl\}thiophen-2-yl]-6-(5-bromothiophen-2-yl)-2,5-}

bis(2-ethylhexyl)-pyrrolo[3,4-c]pyrrole-1,4-dione (7d). This compound was prepared by a method similar to that of 7a, using $6 \mathbf{d}(1.55 \mathrm{~g}, 2.10 \mathrm{mmol})$ and NBS $(0.41 \mathrm{~g}, 2.30 \mathrm{mmol})$. Compound 7d was obtained as a dark violet solid (yield $=1.05 \mathrm{~g}, 61 \%) .{ }^{1} \mathrm{H}$ NMR $(400 \mathrm{MHz}$, $\left.\mathrm{CDCl}_{3}\right): \delta 8.89(\mathrm{~d}, J=4.4 \mathrm{~Hz}, 1 \mathrm{H}), 8.70(\mathrm{~d}, J=4.4 \mathrm{~Hz}, 1 \mathrm{H}), 8.05(\mathrm{~s}, 2 \mathrm{H}), 7.85(\mathrm{~s}, 1 \mathrm{H}), 7.58(\mathrm{~d}, J$ $=4.0 \mathrm{~Hz}, 1 \mathrm{H}), 7.25(\mathrm{~d}, J=4.0 \mathrm{~Hz}, 1 \mathrm{H}), 4.08-3.95(\mathrm{~m}, 4 \mathrm{H}), 1.91-1.84(\mathrm{~m}, 2 \mathrm{H}), 1.40-1.28(\mathrm{~m}, 16 \mathrm{H})$, 0.92-0.85 (m, 12H). MS (MALDI-TOF): $\mathrm{m} / z$ calcd $814.17[M]^{+}$; found, 813.89.

Compound 1. To a mixture of 7a $(0.73 \mathrm{~g}, 1.05 \mathrm{mmol})$ and $\mathbf{8}(0.41 \mathrm{~g}, 0.45 \mathrm{mmol})$ in dry DMF $(40 \mathrm{~mL})$ was added $\mathrm{Pd}\left(\mathrm{PPh}_{3}\right)_{4}(0.02 \mathrm{~g}, 0.02 \mathrm{mmol})$. The mixture was stirred for $17 \mathrm{~h}$ at $85{ }^{\circ} \mathrm{C}$. After cooling to room temperature, the reaction mixture was added into water and then extracted with chloroform. The combined organic layers were washed with water and dried over anhydrous 
$\mathrm{Na}_{2} \mathrm{SO}_{4}$. After filtration and evaporation, the product was purified by silica gel column chromatography (eluent: hexane/chloroform $=7: 3, \mathrm{v} / \mathrm{v}$ ), recrystallized from chloroform/methanol, and dried under vacuum to afford $\mathbf{1}$ as a dark violet solid. This compound was further purified by recycling preparative gel permeation chromatography (GPC; eluent: chloroform) prior to use $($ yield $=0.84 \mathrm{~g}, 97 \%){ }^{1} \mathrm{H} \mathrm{NMR}\left(400 \mathrm{MHz}, \mathrm{CDCl}_{3}\right): \delta 8.99(\mathrm{~d}, J=4.3 \mathrm{~Hz}, 2 \mathrm{H}), 8.94(\mathrm{~d}, J=4.0$ $\mathrm{Hz}, 2 \mathrm{H}), 7.68(\mathrm{~s}, 2 \mathrm{H}), 7.57(\mathrm{dd}, J=8.5,5.3 \mathrm{~Hz}, 4 \mathrm{H}), 7.38(\mathrm{~d}, J=3.3 \mathrm{~Hz}, 2 \mathrm{H}), 7.32(\mathrm{~d}, J=4.3 \mathrm{~Hz}$, 2H), $7.30(\mathrm{~d}, J=4.3 \mathrm{~Hz}, 2 \mathrm{H}), 7.05(\mathrm{t}, J=8.5 \mathrm{~Hz}, 4 \mathrm{H}), 6.99(\mathrm{~d}, 2 \mathrm{H}), 4.07-3.92(\mathrm{~m}, 8 \mathrm{H}), 2.95(\mathrm{~d}, J$ $=7.0 \mathrm{~Hz}, 4 \mathrm{H}), 1.96-1.85(\mathrm{~m}, 4 \mathrm{H}), 1.82-1.73(\mathrm{~m}, 2 \mathrm{H}), 1.52-1.21(\mathrm{~m}, 48 \mathrm{H}), 1.05-0.81(\mathrm{~m}, 36 \mathrm{H})$. ${ }^{13} \mathrm{C}$ NMR $\left(100 \mathrm{MHz}, \mathrm{CDCl}_{3}\right): \delta 161.73,161.36,161,28,148.21,146.30,142.04,139.22,138,93$, $137.36,136.93,136.87,136.72,136.48,129.30,128.92,128.75,128.10,127.66,127.58,125.81$, $125.54,124.17,123.59,120.36,116.17,115.95,108.28,108.14,46.02,45.92,41.45,39.43,39.38$, $34.53,32.72,30.45,30.39,29.03,28.60,25.82,23.18,23.14,14.28,14.12,10.94,1.68,10.59$. MS (MALDI-TOF): $\mathrm{m} / \mathrm{z}$ calcd $1810.74[M]^{+}$; found, 1811.27. Anal. calcd (\%) for $\mathrm{C}_{106} \mathrm{H}_{124} \mathrm{~F}_{2} \mathrm{~N}_{4} \mathrm{O}_{4} \mathrm{~S}_{8}$ : C 70.24, H 6.90, N 3.09; found: C 70.36, H 6.97, N 3.27.

Compound 2. This compound was prepared by a method similar to that of $\mathbf{1}$, using $\mathbf{7 b}(0.69$ g, $0.92 \mathrm{mmol}), 8(0.40 \mathrm{~g}, 0.44 \mathrm{mmol})$, and $\mathrm{Pd}\left(\mathrm{PPh}_{3}\right)_{4}(0.09 \mathrm{~g}, 0.08 \mathrm{mmol})$. Compound 2 was obtained as a dark violet solid, and further purified by recycling preparative GPC (eluent: chloroform) prior to use (yield $=0.26 \mathrm{~g}, 31 \%) .{ }^{1} \mathrm{H}$ NMR (400 MHz, $\left.\mathrm{CDCl}_{3}\right): \delta 9.01(\mathrm{~d}, J=4.3$ $\mathrm{Hz}, 2 \mathrm{H}), 8.93(\mathrm{~d}, J=4.0 \mathrm{~Hz}, 2 \mathrm{H}), 7.71(\mathrm{t}, J=4.3 \mathrm{~Hz}, 6 \mathrm{H}), 7.61$ (d, $J=8.0 \mathrm{~Hz}, 4 \mathrm{H}), 7.47(\mathrm{~d}, J=$ $4.0 \mathrm{~Hz}, 2 \mathrm{H}), 7.38(\mathrm{~d}, J=3.5 \mathrm{~Hz}, 2 \mathrm{H}), 7.33(\mathrm{~d}, J=4.0 \mathrm{~Hz}, 2 \mathrm{H}), 6.98(\mathrm{~d}, J=3.3 \mathrm{~Hz}, 2 \mathrm{H}), 4.08-$ $3.93(\mathrm{~m}, 8 \mathrm{H}), 2.95(\mathrm{~d}, J=6.8 \mathrm{~Hz}, 4 \mathrm{H}), 1.96-1.84(\mathrm{~m}, 4 \mathrm{H}), 1.82-1.74(\mathrm{~m}, 2 \mathrm{H}), 1.48-1.22(\mathrm{~m}, 48 \mathrm{H})$, 1.05-0.80 (m, 36H). ${ }^{13} \mathrm{C}$ NMR (100 MHz, $\left.\mathrm{CDCl}_{3}\right): \delta 161.31,160.99,146.79,146.30,142.23$, 139.35, 138.83, 137.26, 136.50, 136.44, 136.11, 130.19, 129.94, 129.87, 128.47, 128.10, 125.83 , $125.77,125.62,125.51,125.26,125.19,123.46,122.48,120.31,108.51,108.01,45.94,45.85$, $41.38,39.42,34.54,32.70,30.39,30.29,29.02,28.53,28.47,25.76,23.72,23.57,23.21,23.17$, 14.27, 14.17, 14.14, 10.92, 10.62, 10.50. MS (MALDI-TOF): $\mathrm{m} / z$ calcd $1910.73[\mathrm{M}]^{+}$; found, 1911.93. Anal. calcd (\%) for $\mathrm{C}_{108} \mathrm{H}_{124} \mathrm{~F}_{6} \mathrm{~N}_{4} \mathrm{O}_{4} \mathrm{~S}_{8}$ : C 67.82, H 6.53, N 2.93; found: C 67.76, H 6.51, N 2.89 . 
Compound 3. This compound was prepared by a method similar to that of $\mathbf{1}$, using $7 \mathbf{c}(0.66$ g, $0.92 \mathrm{mmol}), 8(0.40 \mathrm{~g}, 0.44 \mathrm{mmol})$, and $\mathrm{Pd}\left(\mathrm{PPh}_{3}\right)_{4}(0.02 \mathrm{~g}, 0.02 \mathrm{mmol})$. Compound 3 was obtained as a dark violet solid, and further purified by recycling preparative GPC (eluent: chloroform) prior to use (yield $=0.72 \mathrm{~g}, 89 \%) .{ }^{1} \mathrm{H}$ NMR $\left(400 \mathrm{MHz}, \mathrm{CDCl}_{3}\right): \delta 9.03(\mathrm{~d}, J=4.3 \mathrm{~Hz}$, 2H), $8.92(\mathrm{~d}, J=4.0 \mathrm{~Hz}, 2 \mathrm{H}), 7.71-7.69(2 \mathrm{H}), 7.41-7.39(2 \mathrm{H}), 7.38(\mathrm{~d}, J=3.3 \mathrm{~Hz}, 2 \mathrm{H}), 7.33(\mathrm{~d}$, $J=4.0 \mathrm{~Hz}, 2 \mathrm{H}), 7.11(\mathrm{~d}, J=6.3 \mathrm{~Hz}, 4 \mathrm{H}), 6.98(\mathrm{~d}, J=3.5 \mathrm{~Hz}, 2 \mathrm{H}), 6.71(\mathrm{t}, J=8.8 \mathrm{~Hz}, 2 \mathrm{H}), 4.08-$ $3.94(\mathrm{~m}, 8 \mathrm{H}), 2.95(\mathrm{~d}, J=6.8 \mathrm{~Hz}, 4 \mathrm{H}), 1.95-1.85(\mathrm{~m}, 4 \mathrm{H}), 1.82-1.74(\mathrm{~m}, 2 \mathrm{H}), 1.48-1.23(\mathrm{~m}, 48 \mathrm{H})$, 1.05-0.83 (m, 36H). ${ }^{13} \mathrm{C}$ NMR (100MHz, $\left.\mathrm{CDCl}_{3}\right): \delta 164.58,164.44,162.10,161.97,161.33$, $161.09,146.35,145.87,142.42$, 139.54, 138.94, 138.95, 138.40, 137.34, 137.34, 137.30, 136.62, $136.42,129.86,128.69,128.11,125.81,125.53,125.46,123.59,120.45,108.71,108.44,108.17$, $46.06,45.91,41.45,39.46,34.54,32.72,30.44,29.03,28.65,28.56,25.81,23.72,23.20,23.15$, 14.24, 14.13, 14.08, 14.94, 10.94, 10.67, 10.55 MS (MALDI-TOF): $\mathrm{m} / z$ calcd $1846.72[\mathrm{M}]^{+}$; found, 1846.74. Anal. calcd (\%) for $\mathrm{C}_{106} \mathrm{H}_{122} \mathrm{~F}_{4} \mathrm{~N}_{4} \mathrm{O}_{4} \mathrm{~S}_{8}$ : C 68.87, H 6.65, N 3.03; found: C 68.95, H 6.64, N 3.01.

Compound 4. This compound was prepared by a method similar to that of $\mathbf{1}$, using $\mathbf{7 d}(0.82$ $\mathrm{g}, 1.01 \mathrm{mmol}), 8(0.45 \mathrm{~g}, 0.50 \mathrm{mmol})$, and $\mathrm{Pd}\left(\mathrm{PPh}_{3}\right)_{4}(0.05 \mathrm{~g}, 0.04 \mathrm{mmol})$. Compound 4 was obtained as a dark violet solid, and further purified by recycling preparative GPC (eluent: chloroform) prior to use (yield $=0.62 \mathrm{~g}, 61 \%) .{ }^{1} \mathrm{H}$ NMR $\left(400 \mathrm{MHz}, \mathrm{CDCl}_{3}\right): \delta 9.11(\mathrm{~d}, J=4.0 \mathrm{~Hz}$, 2H), $8.94(\mathrm{~d}, J=4.3 \mathrm{~Hz}, 2 \mathrm{H}), 7.91(\mathrm{~s}, 4 \mathrm{H}), 7.69(\mathrm{~s}, 2 \mathrm{H}), 7.63(\mathrm{~s}, 2 \mathrm{H}), 7.45(\mathrm{~d}, J=4.0 \mathrm{~Hz}, 2 \mathrm{H})$, $7.37(\mathrm{~d}, J=3.3 \mathrm{~Hz}, 2 \mathrm{H}), 7.29(\mathrm{~d}, J=4.0 \mathrm{~Hz}, 2 \mathrm{H}), 6.99(\mathrm{~d}, J=3.5 \mathrm{~Hz}, 2 \mathrm{H}), 4.10-3.91(\mathrm{~m}, 8 \mathrm{H})$, $2.97(\mathrm{~d}, J=6.8 \mathrm{~Hz}, 4 \mathrm{H}), 1.96-1.77(\mathrm{~m}, 6 \mathrm{H}), 1.54-1.24(\mathrm{~m}, 48 \mathrm{H}), 1.08-0.82(\mathrm{~m}, 36 \mathrm{H}) .{ }^{13} \mathrm{C} \mathrm{NMR}$ $\left(100 \mathrm{MHz}, \mathrm{CDCl}_{3}\right): \delta 161.33,160.95,146.39,144.16,142.76,139.96,138.94,137.74,137.30$, $136.53,136.33,134.91,132.69,132.69,132.36,130.55,128.65,128.09,125.96,125.55,125.02$, $124.27,123.58,121.55,120.57,109.03,108.04,45.99,45.92,41.42,39.60,39.41,39.38,34.51$, $32.66,30.53,30.39,29.01,28.65,28.53,28.51,25.73,23.69,23.15,14.27,14.15,13.96,10.91$, 10.69, 10.62, 10.53. MS (MALDI-TOF): $\mathrm{m} / z$ calcd $2046.70[M]^{+}$; found, 2047.81. Anal. calcd (\%) for $\mathrm{C}_{110} \mathrm{H}_{122} \mathrm{~F}_{12} \mathrm{~N}_{4} \mathrm{O}_{4} \mathrm{~S}_{8}$ : C 64.49, $\mathrm{H}$ 6.00, N 2.73; found: C 64.60, H 5.99, N 2.73. 


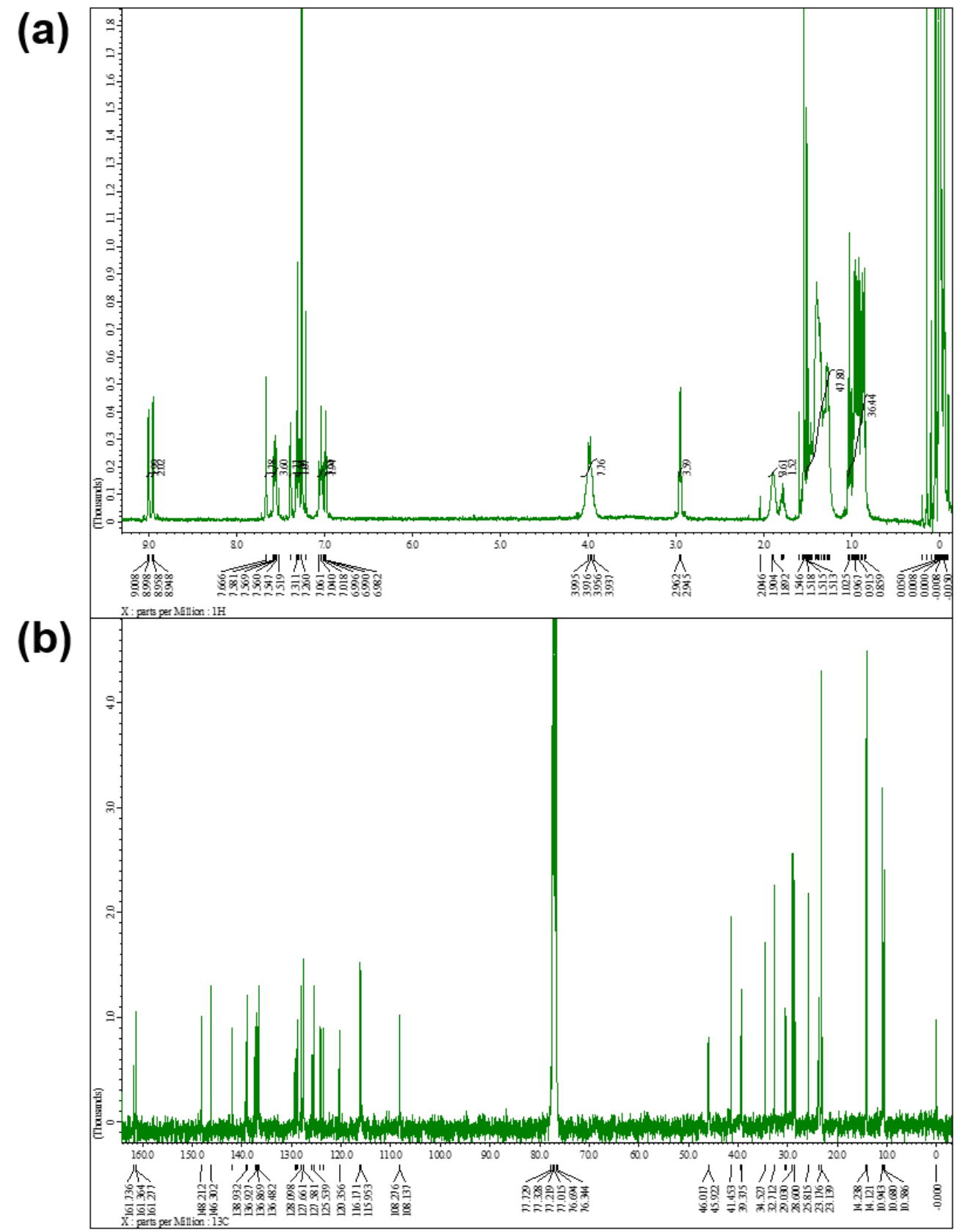

Figure S1. (a) ${ }^{1} \mathrm{H}$ and (b) ${ }^{13} \mathrm{C}\left\{{ }^{1} \mathrm{H}\right\}$ NMR spectra of $\mathbf{1}$ in $\mathrm{CDCl}_{3}$. 

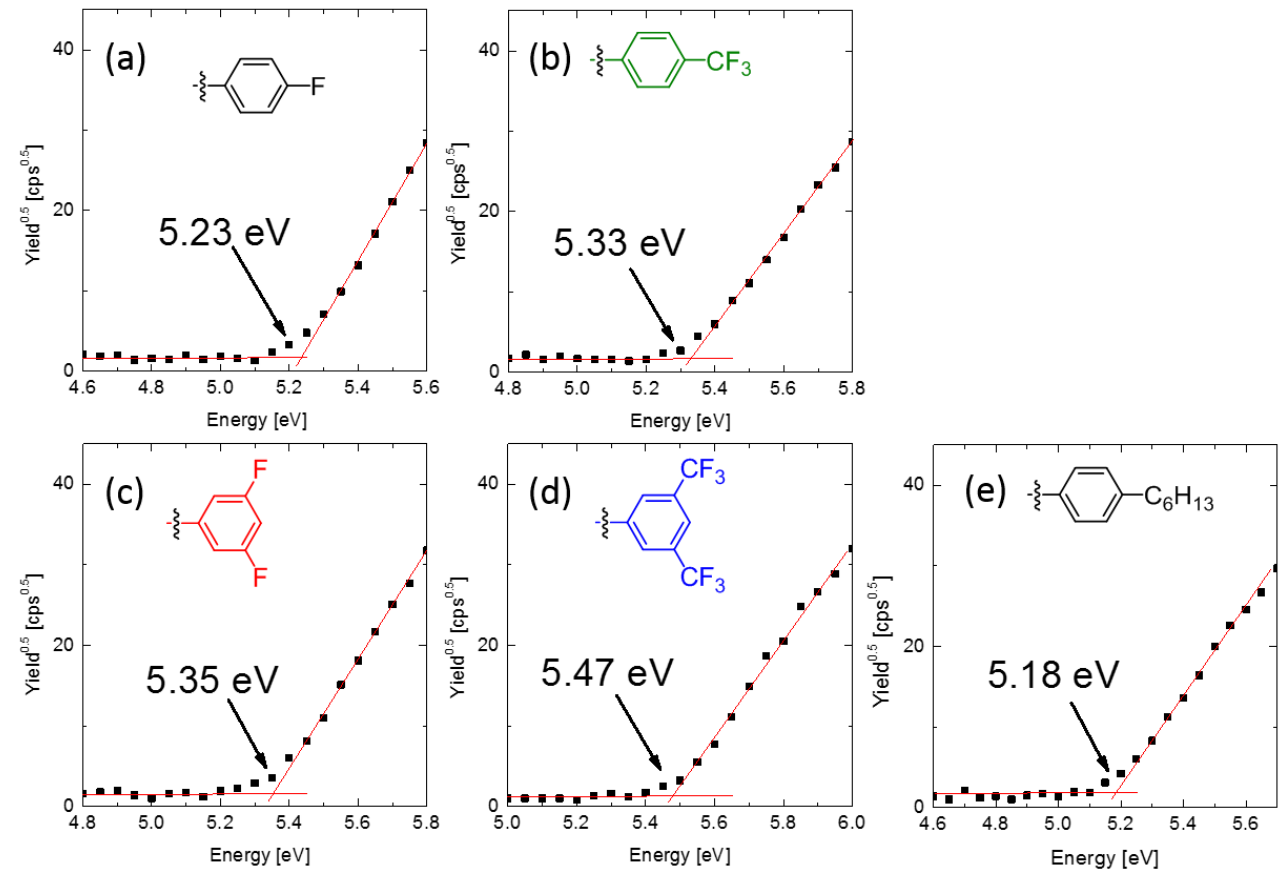

Figure S2. Photoelectron yield spectra of thin films of (a) 1, (b) 2, (c) 3, (d) 4, and (e) BDT-DPP on silicon wafers.

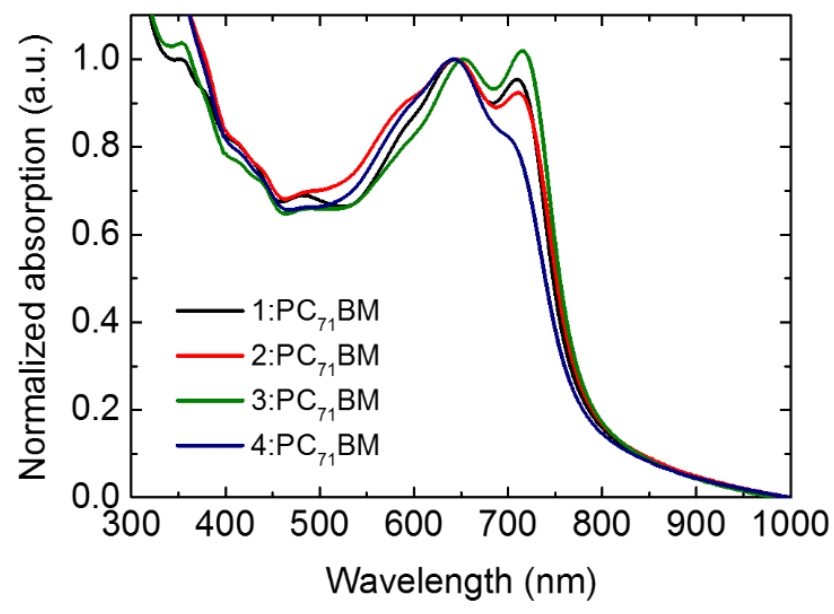

Figure S3. UV-vis absorption spectra of $\mathbf{1 - 4}$ blended with $\mathrm{PC}_{71} \mathrm{BM}(1: 1.5, \mathrm{w} / \mathrm{w})$. 
(a)

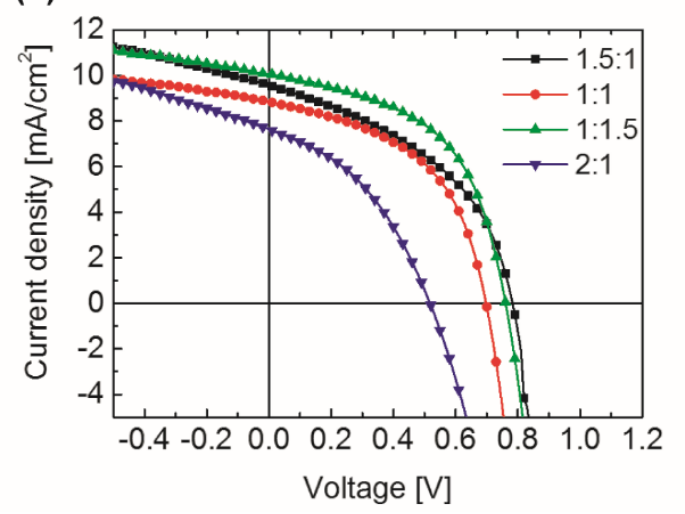

(b)

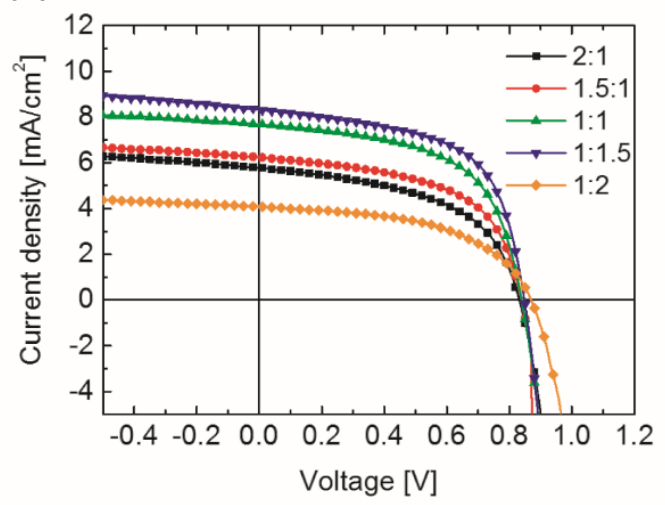

Figure S4. $J-V$ characteristics under one sun illumination for OSCs based on BHJ blends of (a) 1 and (b) 3 with $\mathrm{PC}_{71} \mathrm{BM}$ at different donor/acceptor blend ratios with 0.5 vol\% of 1 chloronaphthalene as a processing additive.

Table S1. Photovoltaic parameters for OSCs based on BHJ blends of $\mathbf{1}$ and $\mathbf{3}$ (donor) with $\mathrm{PC}_{71} \mathrm{BM}$ (acceptor) at different donor/acceptor blend ratios under one sun illumination.

\begin{tabular}{cccccc}
\hline Donor & Blend ratio & $J_{\mathrm{sc}}\left(\mathrm{mA} \mathrm{cm}^{-2}\right)$ & $V_{\mathrm{oc}}(\mathrm{V})$ & $\mathrm{FF}(\%)$ & $\mathrm{PCE}(\%)$ \\
\hline $\mathbf{1}$ & $1.5: 1$ & 9.6 & 0.78 & 44 & 3.3 \\
& $1: 1$ & 8.8 & 0.70 & 50 & 3.1 \\
& $\mathbf{1 : 1 . 5}$ & $\mathbf{1 0 . 1}$ & $\mathbf{0 . 7 6}$ & $\mathbf{5 2}$ & $\mathbf{4 . 0}$ \\
& $1: 2$ & 7.6 & 0.52 & 40 & 1.6 \\
\hline $\mathbf{3}$ & $2: 1$ & 5.8 & 0.83 & 52 & 2.5 \\
& $1.5: 1$ & 6.3 & 0.84 & 56 & 2.9 \\
& $1: 1$ & 7.7 & 0.84 & 58 & 3.7 \\
& $\mathbf{1 : 1 . 5}$ & $\mathbf{8 . 3}$ & $\mathbf{0 . 8 5}$ & $\mathbf{6 0}$ & $\mathbf{4 . 2}$ \\
& $1: 2$ & 4.8 & 0.84 & 44 & 1.8 \\
\hline
\end{tabular}




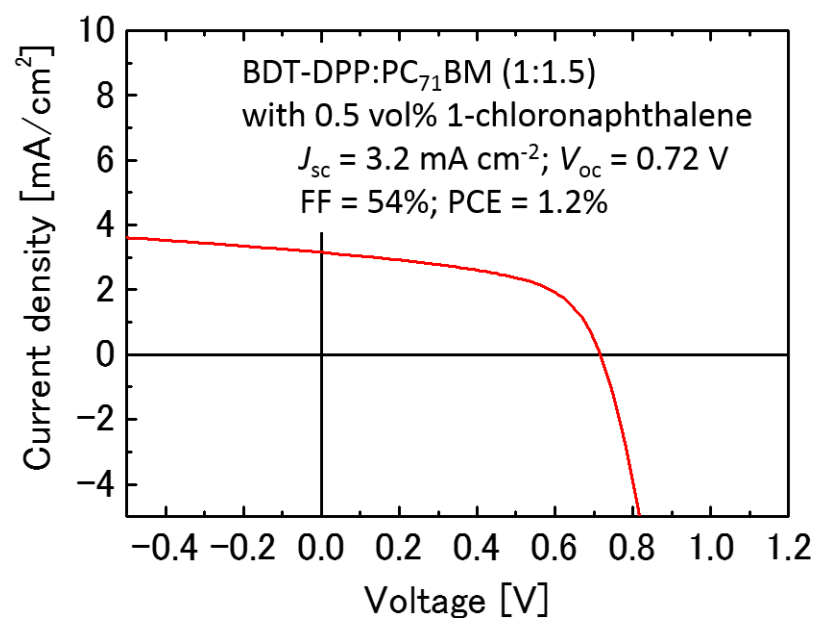

Figure S5. $\quad J-V$ characteristics under one sun illumination for an OSC based on a BHJ blend of BDT-DPP and $\mathrm{PC}_{71} \mathrm{BM}(1: 1.5, \mathrm{v} / \mathrm{v})$ with $0.5 \mathrm{vol} \%$ of 1 -chloronaphthalene as a processing additive.

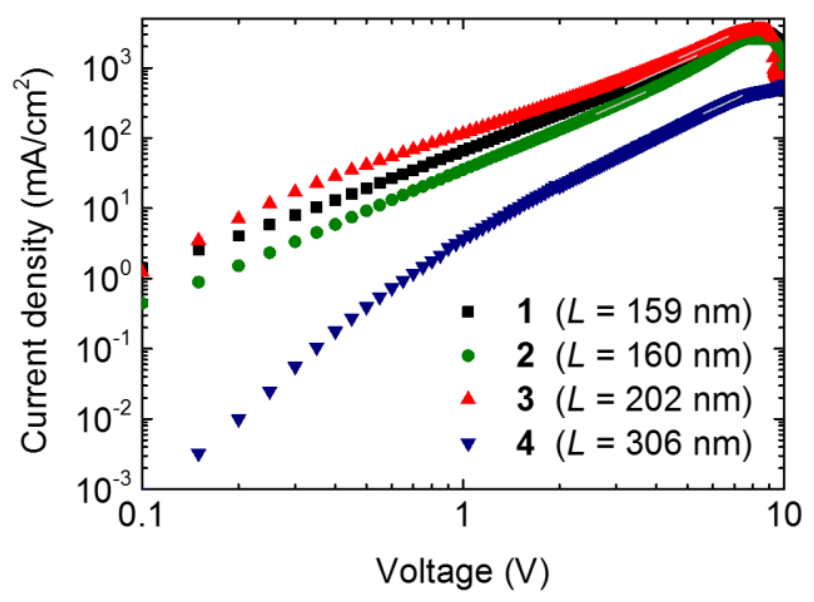

Figure S6. $J-V$ characteristics of hole-only devices using pristine neat films of $\mathbf{1 - 4}$ with device structures of ITO/PEDOT:PSS/donor/ $\mathrm{MoO}_{3} / \mathrm{Ag}$. 